OPEN ACCESS

Edited by:

Guiju Sun,

Southeast University, China

Reviewed by:

Lei Shi,

Georgia State University,

United States

Honglin Dong,

Coventry University,

United Kingdom

Wenzhuo Zhuang,

Soochow University, China Shuwei Xie,

University of Nebraska Medical Center,

United States

*Correspondence:

Zengli $Y u$

zly@zzu.edu.cn

Specialty section:

This article was submitted to Nutritional Immunology,

a section of the journal

Frontiers in Immunology

Received: 01 March 2021

Accepted: 12 July 2021

Published: 05 August 2021

Citation:

Chen S, Wu X and Yu Z (2021) Juglone Suppresses Inflammation and

Oxidative Stress in Colitis Mice.

Front. Immunol. 12:674341.

doi: 10.3389/fimmu.2021.674341

\section{Juglone Suppresses Inflammation and Oxidative Stress in Colitis Mice}

\author{
Shuai Chen ${ }^{1,2}$, Xin $W u^{2}$ and Zengli $Y u^{1,3^{*}}$ \\ ${ }^{1}$ School of Public Health, Xinxiang Medical University, Xinxiang, China, ${ }^{2}$ Key Laboratory of Agro-ecological Processes in \\ Subtropical Region, Institute of Subtropical Agriculture, Chinese Academy of Sciences, Changsha, China, ${ }^{3}$ School of Public \\ Health, Zhengzhou University, Zhengzhou, China
}

Juglone (JUG), a natural product found in walnut trees and other plants, shows potent antioxidant, antimicrobial, and immunoregulatory activities. However, it remains unknown whether JUG can alleviate ulcerative colitis. This study aims to explore the effect of JUG on dextran sulfate sodium (DSS)-induced colitis in mice. The mice were randomly assigned into three groups: the vehicle group, the DSS group, and the JUG group. The experiments lasted for 17 days; during the experiment, all mice received dimethyl sulfoxide (DMSO, $0.03 \% \mathrm{~V} / \mathrm{v}$ )-containing water, while the mice in the JUG group received DMSO-containing water supplemented with JUG $(0.04 \mathrm{w} / \mathrm{v})$. Colitis was induced by administering DSS (3\% $\mathrm{w} / \mathrm{v}$ ) orally for 10 consecutive days. The results showed that the JUG treatment significantly ameliorated body weight loss and disease activity index and improved the survival probability, colon length, and tissue damage. JUG reversed the DSS-induced upregulation of proinflammatory cytokines, including interleukin (IL)-6, 12, 21, and 23, and tumor necrosis factor-alpha, and anti-inflammatory cytokines, such as IL-10 and transforming growth factor-beta, in the serum of the colitis mice. Additionally, the activation of mitochondrial uncoupling protein 2 and phospho-Nuclear Factor-kappa B p65 and the inhibition of the kelch-like ECH-associated protein 1 and NF-E2-related factor 2 induced by DSS were also reversed under JUG administration. Although the JUG group possessed a similar microbial community structure as the DSS group, JUG enriched potential beneficial microbes such as Lachnospiraceae_NK4A136_group but not pathogens such as Escherichia Shigella, which was dominative in DSS group, at the genus level. In conclusion, our results demonstrated that JUG could be a promising agent for UC prevention to regulate inflammatory cytokines and oxidative stress.

Keywords: polyphenol, juglone, mice, DSS, colitis, inflammation, oxidation, microbiota

\section{INTRODUCTION}

Ulcerative colitis (UC), one of the subtypes of inflammatory bowel disease (IBD), is highly prevalent in North America ( $0.29 \%$ in the USA) and Europe ( $0.51 \%$ in Norway), and it has shown an increasing prevalence in Asia, Africa, and South America (1). UC is a relapsing chronic and rectal intestinal disorder with superficial mucosal inflammation that could damage the inner intestinal 
wall, further leading to ulcerations, bleeding, and abdominal cramps, and pain (2). Although UC pathogenesis remains poorly understood, various lines of evidence have demonstrated that intestinal disorders induce immune-mediated inflammation, pathogen invasions, and inflammatory cell infiltration (2), which could further result in continues oxidative stress, inflammation, gut barrier damage, as well as, gut microbiota dysbiosis $(3,4)$.

Numerous polyphenols that have been reported to inhibit colitis in rodents and humans rely on their antioxidant, cell signaling pathways regulation, anti-inflammation, and antipathogen properties (5). Juglone (JUG) is a natural product found in the fruit husk, roots, bark, and leaves of walnut trees and other plants. JUG shows strong antioxidant properties, such as quenching of ROS, enzyme inhibition, and translation metal ion chelation. And JUG is potent in treating oxidative stress-associated diseases such as Alzheimer's disease, kidney fibrogenesis, and liver fibrogenesis (6). JUG also has anticancer, antimicrobial, and immunoregulatory activities, and it can regulate energy metabolism and cell signaling pathways, such as inhibiting the protein kinase B pathway in prostate cancer cells (6-9). JUG has demonstrated antibacterial activity against pathogens such as Staphylococcus aureus, Candida albicans, and Helicobacter pylori $(10,11)$. Wang et al. reported that JUG inhibited tumor growth and metastasis, myeloid-derived suppressor cell accumulation, and interleukin (IL)-1 $\beta$, IL-6, and tumor necrosis factor (TNF)- $\alpha$ production in colorectal cancer mouse models (12). Thus, we suspect JUG might alleviate colitis. However, little is known about the therapeutic effect and underlying mechanisms of JUG on UC.

This study aims to investigate the effects of JUG on dextran sulfate sodium salt (DSS)-induced colitis in mice. We hypothesize that JUG could inhibit inflammation and oxidative stress and regulate gut microbiota in colitis mice.

\section{MATERIALS AND METHODS}

\section{Animals and Experimental Design}

The C57BL6/J mice (male, 6-8 week-old) were assigned into three groups using a randomized block design by body weight ( $n=8 /$ group): the vehicle group (control group), the DSS group, and the JUG group. The experiment was continued for 17 days. All of the mice received drinking water containing $0.03 \%$ dimethyl sulfoxide (DMSO) (Aladdin, Shanghai, China) during the experiment. The mice of the JUG group received DMSOcontaining water supplemented with $0.04 \mathrm{mg} / \mathrm{ml}$ of JUG (Aladdin, Shanghai, China) for 17 days. Colitis induction was performed by administering 3\% (w/v) DSS (MP Biomedicals, MW 36.000-50.000) in tap water ad libitum for mice in the DSS and JUG groups from day 7 to day 17. The mice in the control group drank regular water (Figure 1A).
A

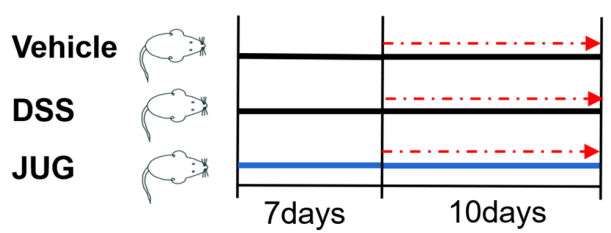

_ drinking water with DMSO drinking water with DMSO and JUG

-.- drinking water with DMSO

C

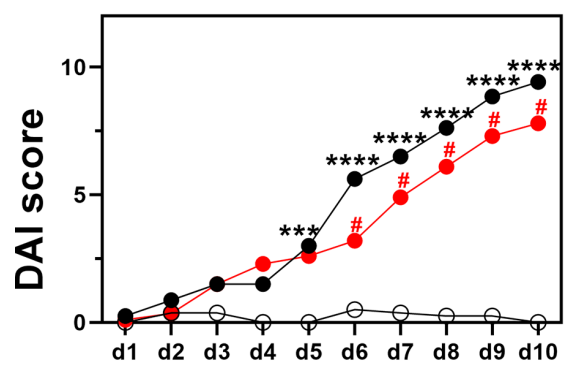

days after onset of colitis
B

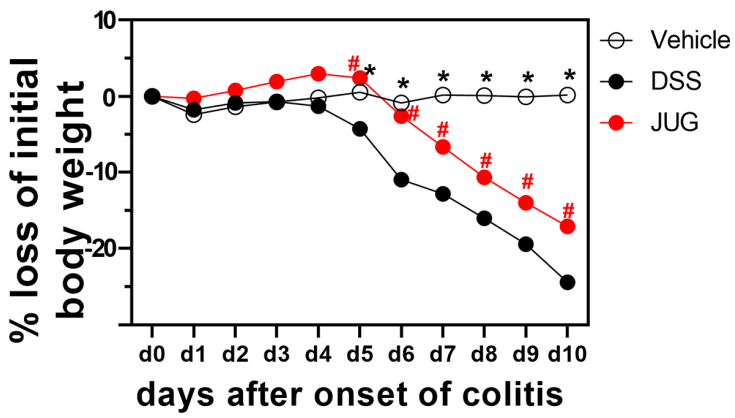

D

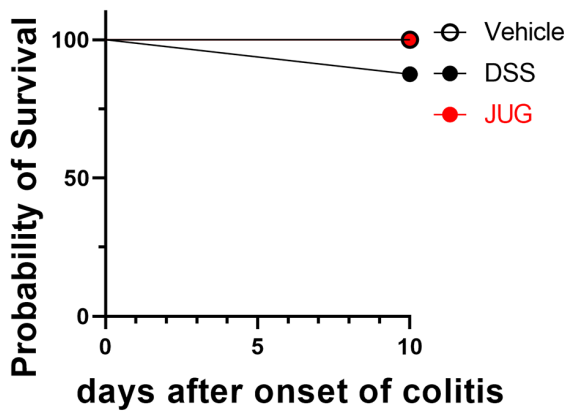

FIGURE 1 | Bodyweight loss, the DAl score, and the survival probability of mice. (A) Schematic showing the treatment of each group. Bodyweight loss percentage (B), DAl score (C), and survival probability (D) during the DSS administration. Two-way ANOVA was used to analyze the difference between the DSS group and the vehicle/JUG group. ${ }^{\star} P<0.05,{ }^{\star \star *} P<0.001,{ }^{\star \star \star *} P<0.0001,{ }^{\sharp} P<0.05$. 
Bodyweight loss, stool consistency, bloody stool, and survival were recorded during the induction of colitis according to a standard scoring system (13), as shown in Supplementary Table 1. The sum of scores on weight loss, stool consistency, and bloody stool was calculated and recorded as disease activity index (DAI). Investigators were blinded to the group allocation while collecting data.

On the day 17, the mice were euthanized to collect the colon and colonic content following blood collection. About $0.5 \mathrm{~cm}$ length of colonic tissue was separated and fixed in $10 \%$ buffered formalin following colonic length measurement, and then the rest of colonic tissue was divided triplicately, snap-frozen in liquid nitrogen, and stored at $-80^{\circ} \mathrm{C}$ before further processing. The blood was centrifuged at $3000 \mathrm{rpm}$ for 10 minutes for serum separation.

\section{Histological and Immunohistochemical Analysis}

The colon was dehydrated and embedded in paraffin wax after being fixed in formalin overnight. After being implanted, several sections of 5 um thickness were sliced according to standard methods (14). The sections were used for hematoxylin and eosin staining (H\&E) and mitochondrial uncoupling protein 2 (Ucp1), nuclear factor erythroid 2-related factor 2 (Nrf2), and kelch-like ECH-associated protein (Keap) 1 immunohistochemical analysis. The histopathological damage of colon tissue was examined on light microscopy (Olympus, Tokyo, Japan) ( $\times 200)$ by Image J software (National Institutes of Health, Bethesda, MD, USA). Histopathological index (HI) was assessed based on a semi-quantitative score criterion (Supplementary Table 2), which is the sum of scores on lymph node numbers, ulcerative area, epithelial changes, and inflammatory cell infiltrate.

Tissue sections used for immunohistochemical analysis were deparaffinized and hydrated. Then, the sections were retrieved in the citric acid buffer ( $\mathrm{pH}$ 6.0) in a microwave oven. Furthermore, the tissue slide was treated with $3 \% \mathrm{H} 2 \mathrm{O} 2$ for $25 \mathrm{~min}$ and incubated with primary antibodies against UCP1, Nrf2, Keap1 (1:100; Servicebio Technology Co. Ltd., Wuhan, China) at $4^{\circ} \mathrm{C}$ overnight. After washing out the excess primary antibody, the samples were treated with Alex Fluor 647 conjugated secondary antibody (Cell Signaling Technology, Boston, MA) and DAPI (Thermo Scientific, Rockford, IL) for $1 \mathrm{~h}$ in the dark. The fluorescence was detected by a laser confocal microscope (Carl Zeiss, Germany).

\section{Antioxidant System Analysis in the Serum}

The serum levels of Malondialdehyde (MDA), glutathione peroxidase (GSH-Px), and total anti-oxidative capacity (TAOC) were analyzed, respectively, using assay kits following the manufacturer's instructions (Beyotime Biotechnology, Shanghai, China).

\section{Enzyme-Linked Immunosorbent Assay (ELISA) Assays}

The content of IL-6, 12, 21, 23, TNF- $\alpha$, IL-10, and TGF- $\beta$ in the serum was quantified by commercial ELISA kits (Multi Sciences
Biotech, Hangzhou, China) according to the instruction of the manufacturer. The BioTek MQX200 microplate reader (BioTek Instruments Inc., Winooski, VT, USA) was used to analyze the optical density of colorimetric reaction at $450 \mathrm{~nm}$. The standard curve was processed according to the optical density and the concentration of the standers using the mELISA software (QINMS, Guilin, China).

\section{Western Blot (WB) Assay}

WB was performed following our previous protocol (15). The colon was homogenized in RIPA lysis buffer containing a complete protease and phosphatase inhibitor (Thermo Fisher Scientific, Shanghai, China) and centrifuged at $4^{\circ} \mathrm{C}$ for $20 \mathrm{~min}$. The supernatant was collected to analyze the total protein concentration using a BCA protein assay kit (Beyotime Biotechnology, China). Equal amounts of proteins of each sample were separated on $10 \%$ SDS-PAGE and then transferred to PVDF membrane (Millipore; Billerica, MA, USA). The PVDF membrane was then incubated with specific primary antibodies (Ucp2,p65,p-p65, Nrf2, Keap1, and $\beta$-actin; Cell Signaling Technology, Danvers, MA, USA) at $4^{\circ} \mathrm{C}$ overnight following incubation with $5 \%$ skim milk for $2 \mathrm{~h}$ at room temperature. Subsequently, membranes were washed in TBST triply and incubated with HRP-conjugated secondary antibodies for $1 \mathrm{~h}$ at room temperature. An enhanced chemiluminescence kit (Tanon, Nanjing, China) was used to visualize the band. Carestream Molecular Imaging system (Carestream Health, Inc., USA) was used to analyze and quantify the intensity of protein bands, further to calculate the integrated optical density (IOD).

\section{$16 S$ rRNA Gene Microbiome Analysis}

According to our previous study (16), the Qiagen QIAamp DNA Stool Mini kit (Qiagen, Beijing, China) was used to extract the total genomic DNA of colonic contents. DNA was quantified by a Nanodrop spectrophotometer (Thermo Scientific, New York, USA), and its purity was tested on $1 \%$ agarose gel. Qualified samples were subsequently amplified the V3-V4 region of ribosomal DNA gene using the universal primers $338 \mathrm{~F}\left(5^{\prime}\right.$ ACTCCTACGGGAGGCAGCA-3') and 806R (5' GGACTACHVGGGTWTCTAA T-3') and were then sequenced on an Illumina Miseq platform. Operational taxonomic units (OTUs) clustering was identified at $97 \%$ sequence identity. The Quantitative Insights into Microbial Ecology (QIIME) software was used to perform alphadiversity, Venn analysis, unweighted UniFrac principal coordinate analysis (PCoA), weighted unifrac PCoA, the linear discriminant analysis (LDA) effect size (LEfSe) algorithm, and PICRUSt2 algorithm. All diagram was processed by using $\mathrm{R}$ software (V2.15.3).

\section{Statistical Analysis}

GraphPad Prism 9 (GraphPad Software, California, United States) was used to perform the statistical analysis. The two-way ANOVA or mixed model methods were used to analyze the difference of bodyweight loss and DAI data between the Vehicle or JUG group and the DSS group. The unpaired t-test or Wilcoxon signed-rank test was used for statistical analysis of other data. The results were 
presented as mean \pm standard error of the mean (SEM), and $P$ value $<0.05$ was considered statistically significant.

\section{RESULTS}

\section{JUG Attenuated DSS-Induced Colitis}

The DAI and pathological scores were monitored in colitis mice to assess the role of JUG in colitis. Water supplemented with JUG significantly reversed DSS-induced $(P<0.05)$ weight loss from day 5 (Figure 1B) and also the DAI score increase from day 7 (Figure 1C). However, the probability of survival was similar between the DSS group and the vehicle or JUG group (Figure 1D). JUG also ameliorated $(P<0.05)$ colon shortening (Figure 2A) and reduced $(P<0.05)$ the pathological score $(P<$ 0.05 ) (Figures 2B, C) in colitis mice. These data indicated that JUG ameliorated DSS-induced colitis.

\section{JUG Inhibited DSS-Induced Inflammation}

Serum proinflammatory cytokines were evaluated to test the effects of JUG on DSS-induced inflammation. JUG reversed $(P<$ 0.05 ) the enrichment of IL-6, 12, 21, and 23, and TNF- $\alpha$ induced by DSS (Figures 3A-E), indicating that JUG reversed DSSinduced inflammation. However, the anti-inflammatory cytokines (IL-10 and TGF- $\beta$ ) were also increased in the DSS group, which was also reduced by JUG administration (Figures 3F, G). These results indicated that JUG reversed DSS-induced inflammation.

\section{JUG Mitigated DSS-Induced Oxidative}

The effects of JUG on intestinal oxidative stress were measured by detection of the colonic T-AOC, GSH-Px, and MD. The results showed that colonic T-AOC and MDA were increased and were not affected by JUG (Figure 4). GSH-Px was lower in the DSS group than that of the controls, and JUG also decreased the GSH-Px concentration (Figures 4B). We next evaluated the colonic expression of p-p65, p65, Keap1, Ucp2, and Nrf2 using WB (Figure 5A) and found that JUG reversed the effects of DSS on activating Ucp2 and p-p65 and inhibiting Keap1 and Nrf2 (Figures 5B-E). Immunofluorescence staining was also performed to measure the colonic expression of Ucp2, Keap1, and Nrf2 (Figure 5). The IOD of Ucp2 and Nrf2 were similar between the DSS group and the vehicle or JUG group (Figures 6A, C). The Keap 1 expression agreed with the result of WB (Figure 6B).

\section{JUG Had Limited Effects on the Gut Microbiota of Colitis Mice}

1197 core OTUs of a total of 2813 OTUs were shared among the three groups, while the unique OUTs in the vehicle group, the DSS group, and the JUG group were 165, 410, and 390, respectively (Figure 7A). Lactobacillus and Lachnospiraceae_NK4A136_group were dominant in the vehicle group, while Escherichia-shigella and Lachnospiraceae_NK4A136_group were dominant in the DSS and JUG groups, separately, at the genus level (Figure 7B). The alphadiversity analysis and PcoA showed no difference between the vehicle or the JUG group and the DSS group (Table 1, Figure 7C). The weighted unifrac PCoA demonstrated that the vehicle group was separated from the DSS group (Figure 7D). The LEfSe analysis indicated that Lactobacillus and L. johnsonii were enriched in the vehicle group, while Escherichia Shigella, Escherichia coli, Bacteroides, B. thetaiotaomicron, Clostridium sensu_stricto_1, Parasutterella, and Helicobacter were enriched in colitis mice, could be used as biomarkers of DSS-induced colitis (LDA score $>4$, Figure $7 \mathbf{E}$ ). In addition, We found Paeniclostridium, P. sordellii, and Clostridium sensu_stricto_1 were less enriched in colitis mice admistered by JUG (LDA score $>$ 3.6, Figure 7F). JUG reduced the DSS-activated function of the mammal gut and animal parasites or symbionts (Figures 7G, H). These results demonstrated that JUG had limited effects on DSS-induced gut microbiota shifts.

\section{DISCUSSION}

The pathogenesis of colitis, a globally prevalent disease that risks the health of millions of people, is still not clearly elucidated. New efficiency bioactivators for managing UC still need to be investigated (17). This study found that JUG potently alleviated DSS-induced colitis as indicated by suppressed proinflammatory cytokines and oxidative stress. Additionally, JUG significantly reversed the DSS-induced activation of Ucp2 and p-p65 and the
A

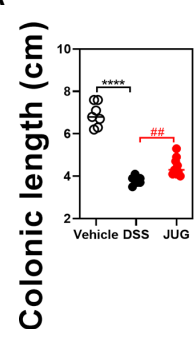

B

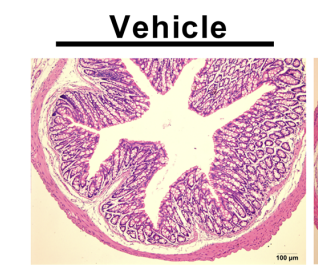

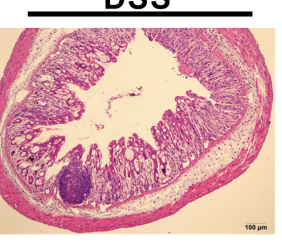

C

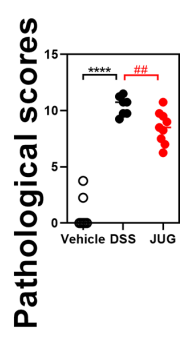

FIGURE 2 | Colon length and pathological scores of mice. (A) Colon length of each mouse in each group. (B) Representative H\&E staining images of the colon $(\times 100)$ and $(\mathbf{C})$ pathological scores from the colonic sections in each group. ${ }^{\star \star \star \star} P<0.0001,{ }^{\# \#} P<0.01$. 
A

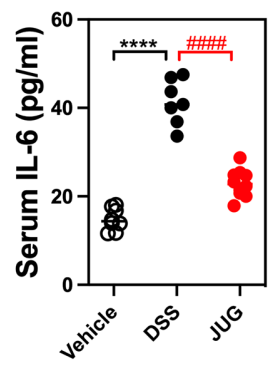

E

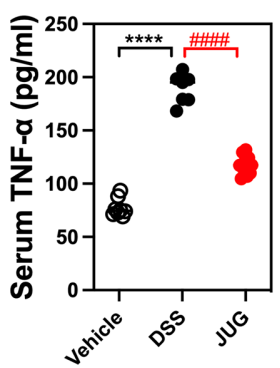

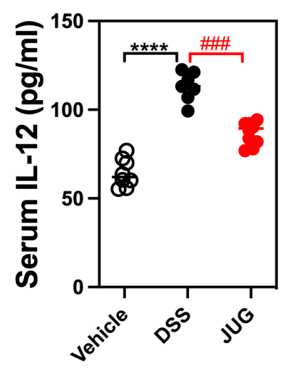

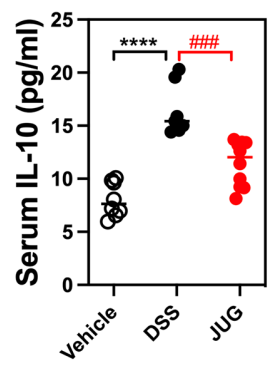

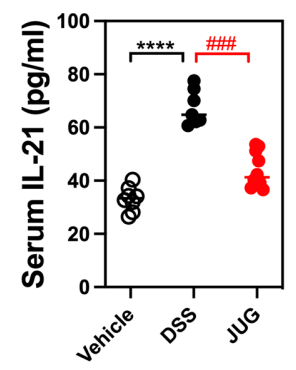

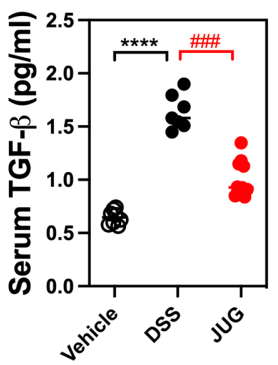

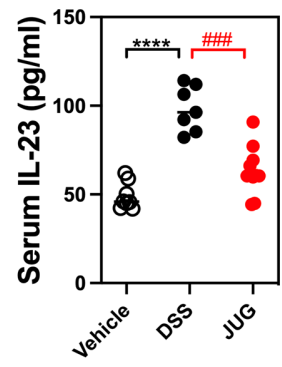

FIGURE 3 | ELISA analysis to test DSS-induced inflammation. ELISA was used to analyze serum cytokines, including IL-6 (A), 12 (B), 21 (C), 23 (D), TNF- $\alpha$ (E),

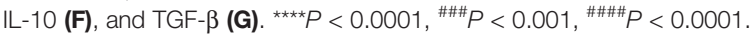

inhibition of Nrf2 signaling. These data may provide new insights into the mechanisms of JUG for controlling symptoms of UC.

Cytokines and cytokines networks are critical regulators in intestinal cellular interactions to modify the gut epithelial barrier function, intestinal immunity and defense, and tissue repair to maintain gut health (18). Proinflammatory cytokines, such as IL-1 $\beta$, TNF- $\alpha$, and IL- 6 driven by intestinal mononuclear phagocytes or epithelial cells, are the inflammatory effectors that initiate intestinal inflammation in UC (19); while IL-12 and IL-23 are regarded as drivers of the pathogenic response to further promote intestinal inflammation (19). Anti-inflammatory cytokines, such as IL-10 and TGF- $\beta$ induced by microbial or metabolites, show vital regulatory roles in immunity tolerance in intestinal inflammation (18). This study found that JUG prevented the elevation of proinflammatory cytokines, including IL-1 $\beta$, IL-6, IL-12, IL-23, and TNF- $\alpha$, induced by DSS treatment. Consistent with our findings, JUG suppressed TNF- $\alpha$, IL- $1 \beta$, and IL- 6 expression in high-fat diet rats (20). JUG was also reported to inhibit TNF- $\alpha$ and Nuclear Factor-kappa B $(\mathrm{NF}-\mathrm{\kappa B})$ production in colonic cancer cells (21). However, there is controversy because DSS enhanced serum IL-10 and TGF- $\beta$
A

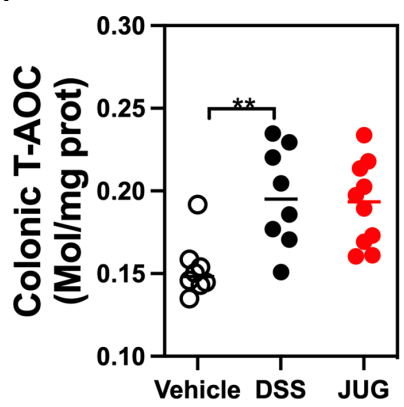

B

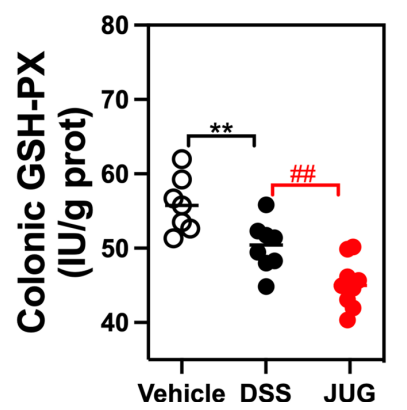

C

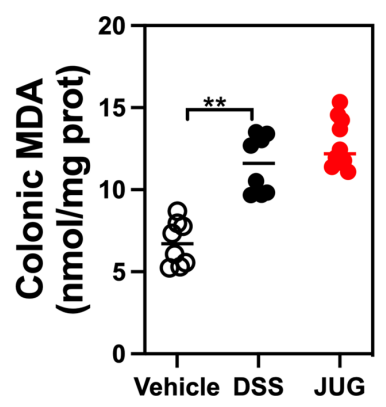

FIGURE 4 | Immunohistochemical examination for analysis of the DSS-induced oxidative damage. The colonic T-AOC (A), MDA (B), and GSH-Px (C) were analyzed by immunohistochemical examination. ${ }^{* *} P<0.01,{ }^{\# \#} P<0.01$. 
A

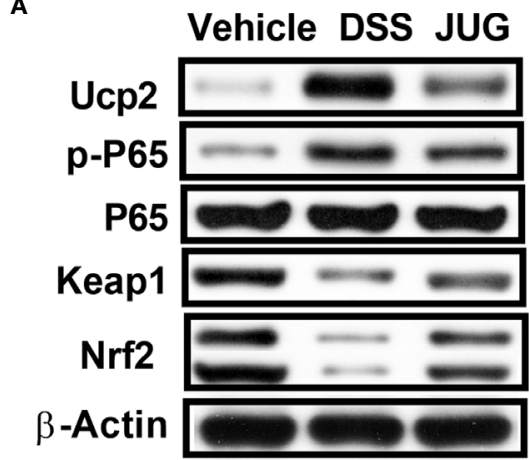

C

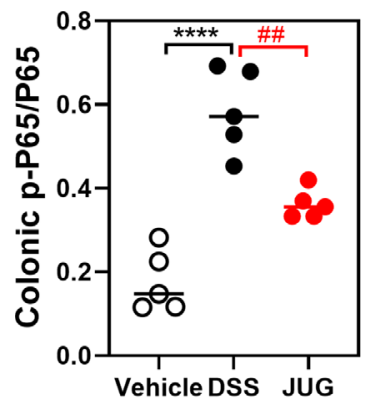

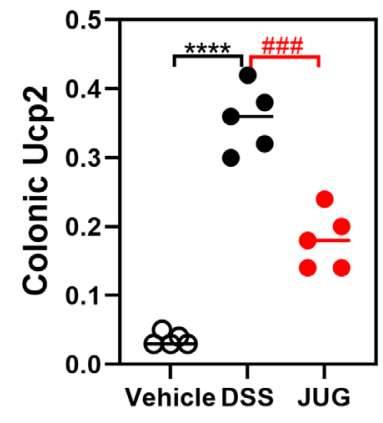

D

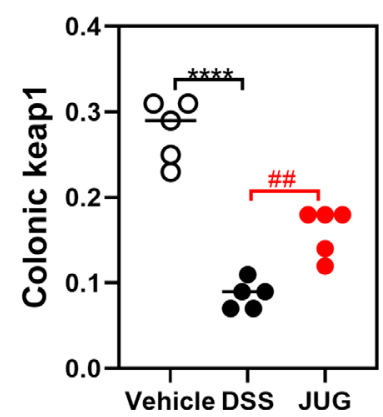

E

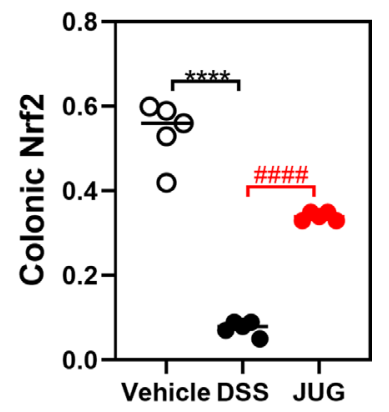

FIGURE 5 | WB for analysis of the DSS-induced oxidative damage. Colonic p-P65, P65, Keap1 Ucp2, and Nrf2 expression on the protein level were analyzed by WB. (A) Representative western blot images of the colonic p-P65, P65, Keap1, Ucp2, NRF2, and $\beta$-Actin proteins. (B-E) Relative densitometric of Ucp2, p-P65/ P65, Keap1, and Nrf2 in the colon. ${ }^{* \star \star *} P<0.0001,{ }^{\# \#} P<0.01,{ }^{\# \# \#} P<0.001,{ }^{\# \# \#} P<0.0001$.

levels while JUG reduced serum levels. As a typical antiinflammatory cytokine produced by various cells (i.e., $\mathrm{B}$ and $\mathrm{T}$ lymphocytes, macrophages, and dendritic cells) in IBD, IL-10 plays a central role in gut homeostasis and colitis prevention (22). Although the IL-10 or IL-10 receptor deficiency mice showed severe gut inflammation in IBD (23), previous studies regarding serum IL-10 in IBD showed inconsistent results; as stated, it decreased, increased, or a similar serum IL-10 was observed in IBD patients $(22,24)$. Additionally, IL-10 supplementation did not achieve the desired effect, and an efficient acceptable IL-10 target therapy is still lacking in humans (25). IL-10 treatment was only efficient in initial colitis but not in established IBD of the mouse model (25). Thus, the role of IL-10 in this study is not clear and requires further study. Moreover, TGF- $\beta$ deficiency induces colitis in mice; however, it also shows proinflammatory properties locally of TGF-b in IBD $(26,27)$. Consistent with our results, Zhao et al. demonstrated that TGF- $\beta 1$ contents increased in the serum and colon of DSS treated rats, while honey, honey polyphenol, and sulfasalazine administration decreased the TGF- $\beta 1$ in both the plasma and colon (26). These results indicated that preserving the cytokines at normal levels may ascribe the effect of JUG for attenuating UC symptoms and further reconstructing the intestinal barrier.
Excess reactive oxygen production is usually observed in chronic intestinal inflammation, indicating that oxidative stress plays a vital role in IBD. Oxidative stress and inflammation facilitate each other, further impairing the structure and function of the intestine at the active stage of colitis $(28,29)$. In UC, NF- $\kappa \mathrm{B}$ pathways were reported significantly activated, resulting in the expression of proinflammatory cytokines. Nrf2 could respond to oxidative stress by modulating cytoprotective gene expressions $(30,31)$. This study found that JUG inhibited Ucp2 and p-p65 while activated Nrf2 in colitis. Accumulating evidence has shown that JUG exerts beneficial effects under inflammatory situations via NF- $\kappa \mathrm{B}$ pathway suppression (20). What's more, 2methoxy-7-acetonyljuglone, a juglone derivative, was also found to possibly increase the nuclear localization of $\mathrm{Nrf} 2$ and its target genes (32). Therefore, the beneficial effect of JUG against colitis may be attributed, at least partially, to the reconstitution of the Nrf2-mediated cellular anti-oxidative system and the suppression of NF- $\mathrm{kB}$ induced inflammation.

Dysbiosis of intestinal flora, commonly characterized by an overgrowth of pathogenic bacteria and a decrease in beneficial bacteria, leads to an improved mucosal immune response and, consequently, inflammation. The Lefse analysis indicated that DSS enriched pathogenic bacteria, such as Clostridium_sensu_stricto_1, and Escherichia Shigella, which were less enriched under JUG 
A
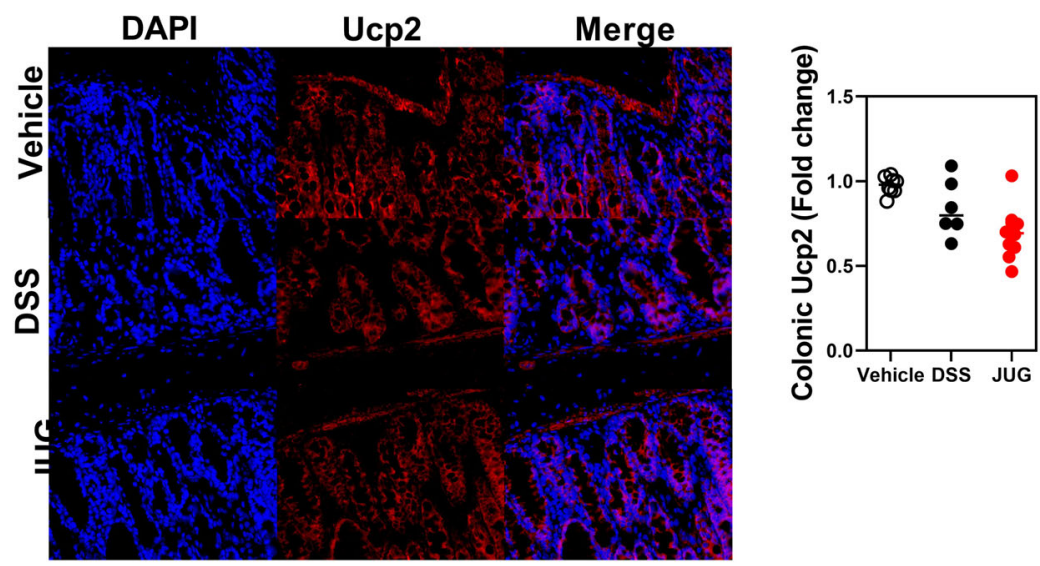

B
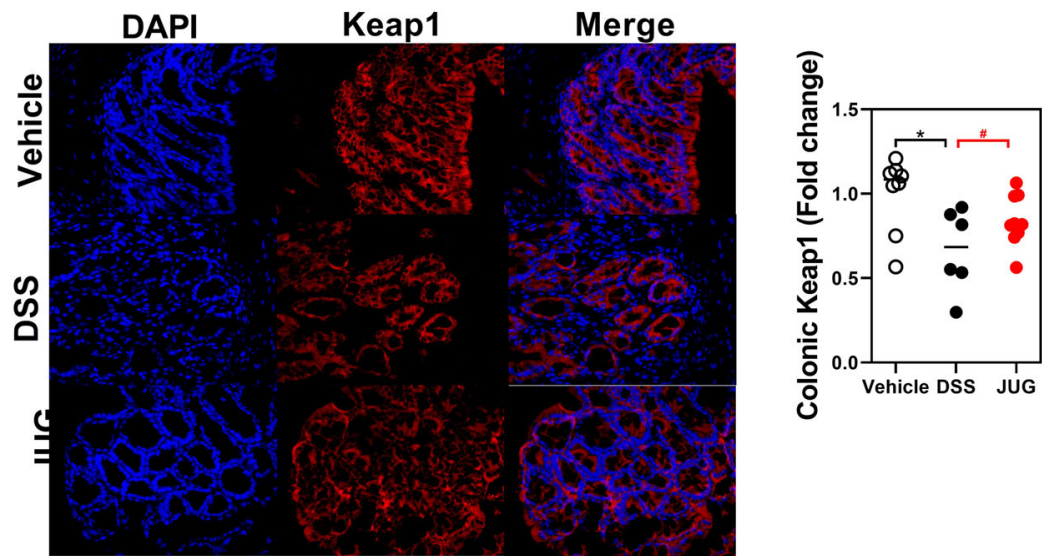

C
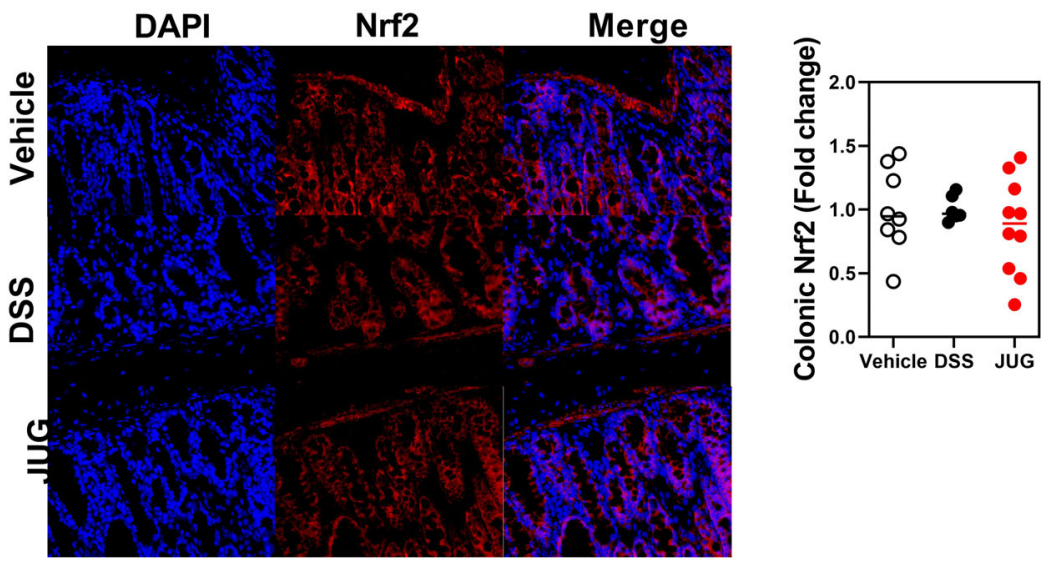

FIGURE 6 | Immunofluorescence staining for analysis of the DSS-induced oxidative damage. Immunofluorescence staining was assigned to measure the colonic expression of Ucp2 (A), Keap1 (B), and Nrf2 (C). ${ }^{*}$ meant $P<0.05$, and ${ }^{\#}$ meant $0.1<P<0.05$, vs. the DSS group.

administration. Escherichia Shigella was the dominative member on the genus level in the DSS group, while Lachnospiraceae_ NK4A136_group genus level was dominant in the JUG-treated colitis mice. The Lachnospiraceae_NK4A136_group has been found to be beneficial to gut health and is regarded as an anti-inflammatory factor due to its production of short-chain fatty acids (33). Similar to our results, Dou et al. reported that sodium butyrate treatment improved colitis and increased the Lachnospiraceae_NK4A136_ group in DSS-induced colitis mice (34). Hence, improvements of the Lachnospiraceae_NK4A136_group and reductions in EscherichiaShigella may partially benefit the restoration of gut microbiota and the reestablishment of intestinal epithelium after JUG treatment. 


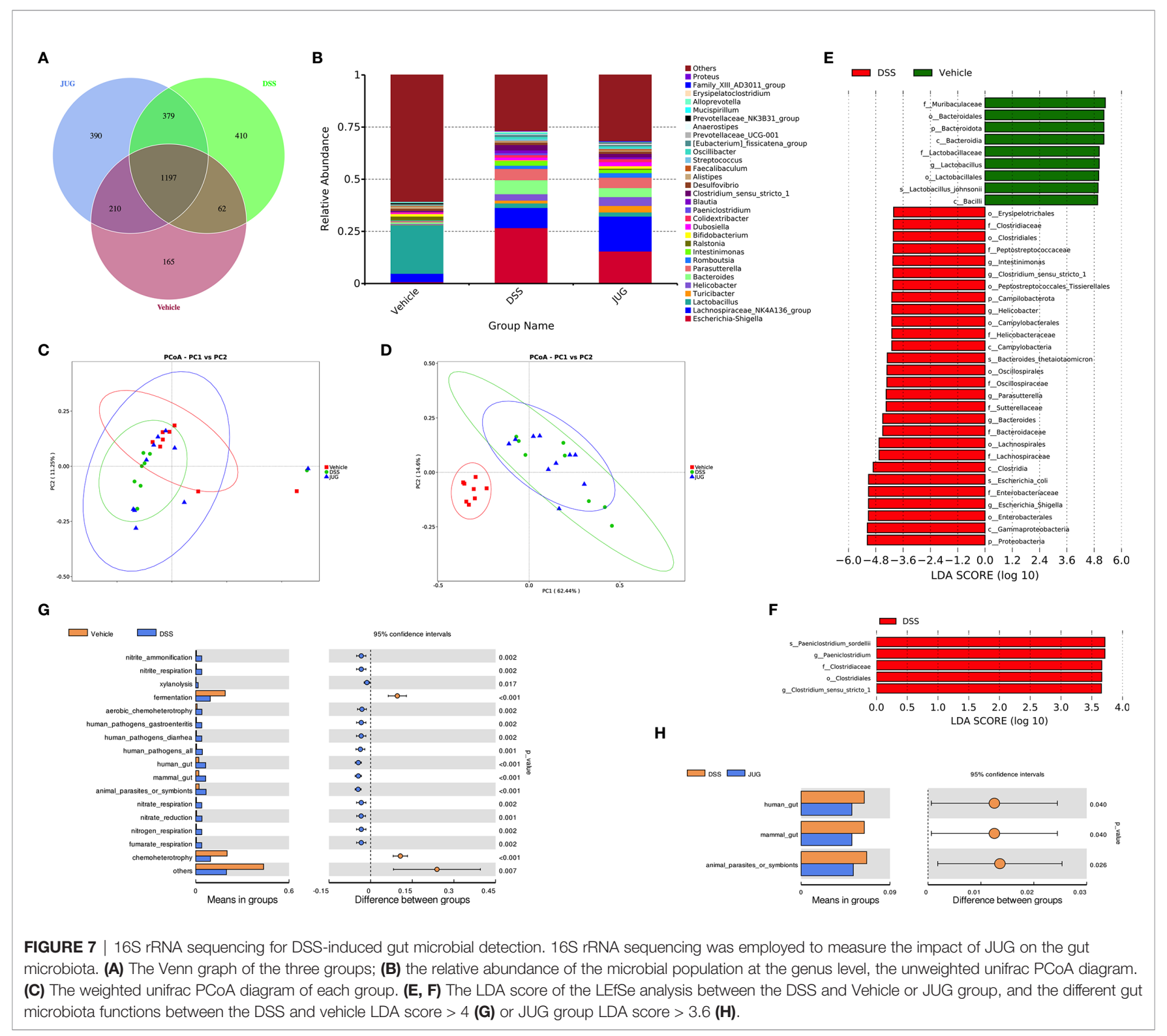

TABLE 1 | Alpha diversity indexes of each group.

\begin{tabular}{lcccc}
\hline & Vehicle & DSS & JUG & SEM \\
\hline Shannon & 5.1379 & 5.2039 & 5.63 & 0.4857 \\
Simpson & 0.9134 & 0.84 & 0.9252 & 0.0617 \\
Chao1 & 616.808 & 765.3958 & 751.7953 & 68.0811 \\
ACE & 632.8906 & 785.9117 & 770.2408 & 69.8313
\end{tabular}

The community richness estimator, including the Chao 1 estimator and the ACE estimator, and the community diversity indexes, including the Shannon index and the Simpson index, were evaluated. An unpaired t-test was used for the analysis between the vehicle/JUG group and the DSS group.

\section{CONCLUSIONS}

In summary, JUG alleviated DSS-induced colitis against colonic inflammation and oxidative stress in DSS-treated mice.
The underlying mechanisms of JUG preventing colitis involved the activation of the Nrf2 and the inhibition of NF- $\mathrm{KB}$ signaling pathways. Moreover, the effects of JUG might also associated with the regulating effect on gut microbiota such 
as Lachnospiraceae_NK4A136_group and Escherichia Shigella. These findings enhance our understanding of the mechanisms of JUG on colitis.

\section{DATA AVAILABILITY STATEMENT}

The original contributions presented in the study are included in the article/Supplementary Material. Further inquiries can be directed to the corresponding author.

\section{ETHICS STATEMENT}

The animal study was reviewed and approved by the Animal Welfare Committee of the Institute of Subtropical Agriculture, Chinese Academy of Sciences.

\section{AUTHOR CONTRIBUTIONS}

SC and ZY designed the experiment. SC and XW performed the experiment, analyzed the data, and prepared tables and figures.

\section{REFERENCES}

1. Ng SC, Shi HY, Hamidi N, Underwood FE, Tang W, Benchimol EI, et al. Worldwide Incidence and Prevalence of Inflammatory Bowel Disease in the 21st Century: A Systematic Review of Population-Based Studies. Lancet (2017) 390(10114):2769-78. doi: 10.1016/S0140-6736(17)32448-0

2. Eisenstein M. Biology: A Slow-Motion Epidemic. Nature (2016) 540(7634): S98-9. doi: 10.1038/540S98a

3. Tian T, Wang Z, Zhang J. Pathomechanisms of Oxidative Stress in Inflammatory Bowel Disease and Potential Antioxidant Therapies. Oxid Med Cell Longev (2017) 2017:4535194. doi: 10.1155/2017/4535194

4. Hooper LV, Littman DR, Macpherson AJ. Interactions Between the Microbiota and the Immune System. Science (2012) 336(6086):1268-73. doi: $10.1126 /$ science. 1223490

5. Martin DA, Bolling BW. A Review of the Efficacy of Dietary Polyphenols in Experimental Models of Inflammatory Bowel Diseases. Food Funct (2015) 6 (6):1773-86. doi: 10.1039/c5fo00202h

6. Ahmad T, Suzuki YJ. Juglone in Oxidative Stress and Cell Signaling. Antioxid (Basel) (2019) 8(4):91. doi: 10.3390/antiox8040091

7. Saling SC, Comar JF, Mito MS, Peralta RM, Bracht A. Actions of Juglone on Energy Metabolism in the Rat Liver. Toxicol Appl Pharmacol (2011) 257 (3):319-27. doi: 10.1016/j.taap.2011.09.004

8. Zhang YY, Zhang F, Zhang YS, Thakur K, Zhang JG, Liu Y, et al. Mechanism of Juglone-Induced Cell Cycle Arrest and Apoptosis in Ishikawa Human Endometrial Cancer Cells. J Agric Food Chem (2019) 67(26):7378-89. doi: 10.1021/acs.jafc.9b02759

9. Fang F, Chen S, Ma J, Cui J, Li Q, Meng G, et al. Juglone Suppresses EpithelialMesenchymal Transition in Prostate Cancer Cells via the Protein Kinase B/ Glycogen Synthase Kinase-3beta/Snail Signaling Pathway. Oncol Lett (2018) 16(2):2579-84. doi: 10.3892/ol.2018.8885

10. Kong YH, Zhang L, Yang ZY, Han C, Hu LH, Jiang HL, et al. Natural Product Juglone Targets Three Key Enzymes From Helicobacter Pylori: Inhibition Assay With Crystal Structure Characterization. Acta Pharmacol Sin (2008) 29 (7):870-6. doi: 10.1111/j.1745-7254.2008.00808.x

11. Strugstad M, Despotovski S. A Summary of Extraction, Synthesis, Properties, and Potential Uses of Juglone: A Literature Review. J Ecosystems Manage (2013) 13(3):72-82.
SC and ZY prepared the manuscript. All authors contributed to the article and approved the submitted version.

\section{FUNDING}

This research was supported by Zhongyuan Science and Technology Innovation Leadership Program No.214200510016.

\section{SUPPLEMENTARY MATERIAL}

The Supplementary Material for this article can be found online at: https://www.frontiersin.org/articles/10.3389/fimmu.2021. 674341/full\#supplementary-material

Supplementary Table 1 | DAl score protocol. DAl score was used to evaluate colitis's clinical activity, and it was the total sum of body weight loss score, stool consistency score, and bloody stools score.

Supplementary Table 2 | Pathological score protocol. The pathological score was used to evaluate intestinal inflammation, and it was the total sum scores of lymph node numbers, ulcerative area (\%), epithelial changes, and inflammatory cell infiltrate.

12. Wang H, Zou C, Zhao W, Yu Y, Cui Y, Zhang H, et al. Juglone Eliminates MDSCs Accumulation and Enhances Antitumor Immunity. Int Immunopharmacol (2019) 73:118-27. doi: 10.1016/j.intimp.2019.04.058

13. Kim JJ, Shajib MS, Manocha MM, Khan WI. Investigating Intestinal Inflammation in DSS-Induced Model of IBD. JoVE (J Vis Exp) (2012) 60): e3678. doi: $10.3791 / 3678$

14. Chen S, Wang M, Yin L, Ren W, Bin P, Xia Y, et al. Effects of Dietary Tryptophan Supplementation in the Acetic Acid-Induced Colitis Mouse Model. Food Funct (2018) 9(8):4143-52. doi: 10.1039/c8fo01025k

15. Chen S, Wu X, Duan J, Huang P, Li T, Yin Y, et al. Low-Protein Diets Supplemented With Glutamic Acid or Aspartic Acid Ameliorates Intestinal Damage in Weaned Piglets Challenged With Hydrogen Peroxide. Anim Nutr (2021) 7(2):356-64. doi: 10.1016/j.aninu.2020.12.005

16. Chen S, Wu X, Wang X, Shao YR, Tu Q, Yang HS, et al. Responses of Intestinal Microbiota and Immunity to Increasing Dietary Levels of Iron Using a Piglet Model. Front Cell Dev Biol (2020) 8:603392. doi: 10.3389/fcell.2020.603392

17. Kamm MA. Rapid Changes in Epidemiology of Inflammatory Bowel Disease. Lancet (2017) 390(10114):2741-2. doi: 10.1016/S0140-6736(17)32669-7

18. Friedrich M, Pohin M, Powrie F. Cytokine Networks in the Pathophysiology of Inflammatory Bowel Disease. Immunity (2019) 50(4):992-1006. doi: 10.1016/j.immuni.2019.03.017

19. Chauhan D, Vande Walle L, Lamkanfi M. Therapeutic Modulation of Inflammasome Pathways. Immunol Rev (2020) 297(1):123-38. doi: 10.1111/ imr. 12908

20. Peng X, Nie Y, Wu J, Huang Q, Cheng Y. Juglone Prevents Metabolic Endotoxemia-Induced Hepatitis and Neuroinflammation via Suppressing TLR4/NF-kappaB Signaling Pathway in High-Fat Diet Rats. Biochem Biophys Res Commun (2015) 462(3):245-50. doi: 10.1016/j.bbrc.2015.04.124

21. Seetha A, Devaraj H, Sudhandiran G. Indomethacin and Juglone Inhibit Inflammatory Molecules to Induce Apoptosis in Colon Cancer Cells. J Biochem Mol Toxicol (2020) 34(2):e22433. doi: 10.1002/jbt.22433

22. Marlow GJ, van Gent D, Ferguson LR. Why Interleukin-10 Supplementation Does Not Work in Crohn's Disease Patients. World J Gastroenterol (2013) 19 (25):3931-41. doi: 10.3748/wjg.v19.i25.3931

23. Glocker EO, Kotlarz D, Boztug K, Gertz EM, Schaffer AA, Noyan F, et al. Inflammatory Bowel Disease and Mutations Affecting the Interleukin-10 Receptor. N Engl J Med (2009) 361(21):2033-45. doi: 10.1056/NEJMoa0907206 
24. Melgar S, Yeung MM, Bas A, Forsberg G, Suhr O, Oberg A, et al. OverExpression of Interleukin 10 in Mucosal T Cells of Patients With Active Ulcerative Colitis. Clin Exp Immunol (2003) 134(1):127-37. doi: 10.1046/ j.1365-2249.2003.02268.x

25. Neumann C, Scheffold A, Rutz S. Functions and Regulation of T Cell-Derived Interleukin-10. Semin Immunol (2019) 44:101344. doi: 10.1016/ j.smim.2019.101344

26. Kulkarni AB, Huh CG, Becker D, Geiser A, Lyght M, Flanders KC, et al. Transforming Growth Factor Beta 1 Null Mutation in Mice Causes Excessive Inflammatory Response and Early Death. Proc Natl Acad Sci USA (1993) 90 (2):770-4. doi: 10.1073/pnas.90.2.770

27. Del Zotto B, Mumolo G, Pronio AM, Montesani C, Tersigni R, Boirivant M. TGF- $\beta 1$ Production in Inflammatory Bowel Disease: Differing Production Patterns in Crohn's Disease and Ulcerative Colitis. Clin Exp Immunol (2003) 134(1):120-6. doi: 10.1046/j.1365-2249.2003.02250.x

28. Dudzinska E, Gryzinska M, Ognik K, Gil-Kulik P, Kocki J. Oxidative Stress and Effect of Treatment on the Oxidation Product Decomposition Processes in IBD. Oxid Med Cell Longev (2018) 2018:7918261. doi: 10.1155/2018/7918261

29. Wang Z, Li S, Cao Y, Tian X, Zeng R, Liao DF, et al. Oxidative Stress and Carbonyl Lesions in Ulcerative Colitis and Associated Colorectal Cancer. Oxid Med Cell Longev (2016) 2016:9875298. doi: 10.1155/2016/9875298

30. Qiu S, Li P, Zhao H, Li X. Maresin 1 Alleviates Dextran Sulfate SodiumInduced Ulcerative Colitis by Regulating NRF2 and TLR4/NF-kB Signaling Pathway. Int Immunopharmacol (2020) 78:106018. doi: 10.1016/ j.intimp.2019.106018

31. Jing $M$, Wang $Y, X u$ L. Andrographolide Derivative AL-1 Ameliorates Dextran Sodium Sulfate-Induced Murine Colitis by Inhibiting NF-kappaB and MAPK Signaling Pathways. Oxid Med Cell Longev (2019) 2019:6138723. doi: $10.1155 / 2019 / 6138723$
32. Kim JH, Khalil AAK, Kim HJ, Kim SE, Ahn MJ. 2-Methoxy-7Acetonyljuglone Isolated From Reynoutria Japonica Increases the Activity of Nuclear Factor Erythroid 2-Related Factor-2 Through Inhibition of Ubiquitin Degradation in HeLa Cells. Antioxid (Basel) (2019) 8(9):398. doi: 10.3390/antiox 8090398

33. Wang C, Li W, Wang H, Ma Y, Zhao X, Zhang X, et al. Saccharomyces Boulardii Alleviates Ulcerative Colitis Carcinogenesis in Mice by Reducing TNF-Alpha and IL-6 Levels and Functions and by Rebalancing Intestinal Microbiota. BMC Microbiol (2019) 19(1):246. doi: 10.1186/s12866-019-1610-8

34. Dou X, Gao N, Yan D, Shan A. Sodium Butyrate Alleviates Mouse Colitis by Regulating Gut Microbiota Dysbiosis. Animals (2020) 10(7):1154. doi: 10.3390/ani10071154

Conflict of Interest: The authors declare that the research was conducted in the absence of any commercial or financial relationships that could be construed as a potential conflict of interest.

Publisher's Note: All claims expressed in this article are solely those of the authors and do not necessarily represent those of their affiliated organizations, or those of the publisher, the editors and the reviewers. Any product that may be evaluated in this article, or claim that may be made by its manufacturer, is not guaranteed or endorsed by the publisher.

Copyright (C) 2021 Chen, $W u$ and $Y u$. This is an open-access article distributed under the terms of the Creative Commons Attribution License (CC BY). The use, distribution or reproduction in other forums is permitted, provided the original author(s) and the copyright owner(s) are credited and that the original publication in this journal is cited, in accordance with accepted academic practice. No use, distribution or reproduction is permitted which does not comply with these terms. 